J. Korean Math. Soc. 51 (2014), No. 5, pp. 881-895

http://dx.doi.org/10.4134/JKMS.2014.51.5.881

\title{
REMARKS ON LEVI HARMONICITY OF CONTACT SEMI-RIEMANNIAN MANIFOLDS
}

\author{
Domenico Perrone
}

\begin{abstract}
In a recent paper [10] we introduced the notion of Levi harmonic map $f$ from an almost contact semi-Riemannian manifold $(M, \varphi, \xi$, $\eta, g$ ) into a semi-Riemannian manifold $M^{\prime}$. In particular, we computed the tension field $\tau_{\mathcal{H}}(f)$ for a $C R$ map $f$ between two almost contact semi-Riemannian manifolds satisfying the so-called $\varphi$-condition, where $\mathcal{H}=\operatorname{Ker}(\eta)$ is the Levi distribution. In the present paper we show that the condition $(A)$ of Rawnsley [17] is related to the $\varphi$-condition. Then, we compute the tension field $\tau_{\mathcal{H}}(f)$ for a $C R$ map between two arbitrary almost contact semi-Riemannian manifolds, and we study the concept of Levi pluriharmonicity. Moreover, we study the harmonicity on quasicosymplectic manifolds.
\end{abstract}

\section{Introduction}

As a natural continuation of the ideas in [2], and following the ideas of B. Fuglede (who started the study of the semi-Riemannian case within harmonic map theory, cf. [11] and [1], pp. 427-455), in the recent paper [10] S. Dragomir and the present author introduced the concept of Levi harmonic map $f$ from an almost contact semi-Riemannian manifold $(M, \varphi, \xi, \eta, g)$ into a semi-Riemannian manifold $\left(M^{\prime}, g^{\prime}\right)$, i.e., $C^{\infty}$ solutions of $\tau_{\mathcal{H}}(f) \equiv \operatorname{trace}_{g}\left(\Pi_{\mathcal{H}} \beta_{f}\right)=0$, where $\beta_{f}$ is the second fundamental form of $f$, and $\Pi_{\mathcal{H}} \beta_{f}$ is the restriction of $\beta_{f}$ to the Levi distribution $\mathcal{H}=\operatorname{Ker}(\eta)$. Thus, we studied the Levi harmonicity for CR maps between two almost contact semi-Riemannian manifolds. This is perhaps the most general geometric setting (metrics are but semi-Riemannian and in general the contact condition (2.2) is not satisfied and the underlying almost CR structures are not integrable).

In the study of [10] an important role is played by the notion of $\varphi$-condition (cf. (3.1) in §3), in particular we computed the tension field $\tau_{\mathcal{H}}(f)$ for a $C R$ map

Received December 18, 2012.

2010 Mathematics Subject Classification. 53C43, 53D15, 32V05, 53C25, 53C50, 53C40.

Key words and phrases. almost contact semi-Riemannian manifold, $\varphi$-condition, CR map, invariant submanifold, Levi harmonicity, Levi pluriharmonicity.

Supported by funds of the Universitá del Salento and of the M.I.U.R. (within PRIN 2010-2011) 
$f$ between two almost contact semi-Riemannian manifolds satisfying the the $\varphi$-condition. Moreover, we emphasized that the class of almost contact semiRiemannian manifolds obeying to (3.1) is quite large. For instance, contact semi-Riemannian manifolds, quasi-cosimplectic manifolds and orientable real hypersurfaces in an indefinite Kaehler manifold (with the induced almost contact semi-Riemannian structure) satisfy the $\varphi$-condition. On the other hand, Rawnsley [17] introduced the so-called condition $(A)$ in order to study the harmonicity of $f$-holomorphic maps between an almost Hermitian manifold with coclosed Kaehler form and a Riemannian manifold equipped with a $f$-structure which satisfies condition $(A)$.

In the present paper we show that the condition $(A)$ of Rawnsley is related to the $\varphi$-condition, more precisely (cf. Theorem 3.2): if an almost contact semi-Riemannian structure satisfies the condition $(A)$, then it satisfies the $\varphi$-condition. The converse does not hold. In Section 4 , we compute the tension field $\tau_{\mathcal{H}}(f)$ for a $C R$ map $f: M \rightarrow M^{\prime}$ between two arbitrary almost contact semi-Riemannian manifolds (this result extends Theorem 3.9 of [10]). In Section 5, in analogy with the Kaehlerian case [12], we introduce and study the concept of Levi pluriharmonicity (cf. (5.1)). Of course Levi pluriharmonicity implies Levi harmonicity. In particular we get that if $M$ is an invariant semi-Riemannian submanifold of a contact semi-Riemannian manifold $\bar{M}$, then the inclusion $i: M \rightarrow \bar{M}$ is Levi pluriharmonic and a pseudohermitian map. In the last section, we show that any $\mathrm{CR}$ map $f: M \rightarrow M^{\prime}$ among two quasi-cosymplectic manifolds is Levi pluriharmonic. Moreover, a CR map $f: M \rightarrow M^{\prime}$ among two quasi-cosymplectic manifolds is a harmonic map if and only if $f_{*} \xi$ is a geodesic vector field.

\section{Preliminaries}

\subsection{Contact semi-Riemannian manifolds}

Let $M$ be a real $(2 n+1)$-dimensional $C^{\infty}$ manifold. An almost contact structure $(\varphi, \xi, \eta)$ on $M$ consists of a $(1,1)$-tensor field $\varphi$, a tangent vector field $\xi \in \mathfrak{X}(M)$ (the characteristic, or Reeb, vector field) and a differential 1-form $\eta$ such that

$$
\varphi^{2}=-I+\eta \otimes \xi, \quad \eta(\xi)=1 .
$$

In particular $\varphi(\xi)=0$ and $\eta \circ \varphi=0$. Let $\varepsilon \in\{ \pm 1\}$. Given an almost contact structure $(\varphi, \xi, \eta)$ on $M$ a compatible metric is a semi-Riemannian metric $g$ on $M$ such that

$$
g(\varphi X, \varphi Y)=g(X, Y)-\varepsilon \eta(X) \eta(Y), \quad X, Y \in \mathfrak{X}(M) .
$$

Then $\eta(X)=\varepsilon g(\xi, X)$ and $g(\xi, \xi)=\varepsilon$. Therefore the characteristic vector field $\xi$ is either spacelike or timelike ( $\xi$ is never lightlike). The synthetic object $(\varphi, \xi, \eta, g)$ is an almost contact semi-Riemannian structure. If in addition the contact condition

$$
d \eta=g(\cdot, \varphi \cdot)
$$


is satisfied, then $\eta$ is a contact form, i.e., $\eta \wedge(d \eta)^{n}$ is a volume form on $M$ (and $(\varphi, \xi, \eta, g)$ is referred to as contact semi-Riemannian structure on $M)$. On each contact semi-Riemannian manifold the tensor field $h=(1 / 2) \mathcal{L}_{\xi} \varphi$ (where $\mathcal{L}$ is the Lie derivative) is symmetric and satisfies

$$
\nabla \xi=-\varepsilon \varphi-\varphi \circ h, \quad \nabla_{\xi} \varphi=0, \quad h \circ \varphi+\varphi \circ h=0, \quad h(\xi)=0 .
$$

Here $\nabla$ is the Levi-Civita connection of the semi-Riemannian manifold $(M, g)$. Moreover (by Lemma 4.3 in [6])

$$
\left(\nabla_{X} \varphi\right) Y+\left(\nabla_{\varphi X} \varphi\right) \varphi Y=2 g(X, Y) \xi-\eta(Y)\{\varepsilon X+\varepsilon \eta(X) \xi+h(X)\} .
$$

We may refer to [6], [15], [16] for more information about the geometry of a contact semi-Riemannian manifold.

\subsection{Almost CR structures}

Let $M$ be a real $(2 n+1)$-dimensional manifold. An almost $C R$ structure on $M$ is a complex subbbundle $T_{1,0}(M)$, of complex rank $n$, of the complexified tangent bundle $T(M) \otimes \mathbb{C}$ such that $T_{1,0}(M) \cap T_{0,1}(M)=(0)$, where $T_{0,1}(M)=\overline{T_{1,0}(M)}$ (overbars denote complex conjugates). The integer $n$ is the $C R$ dimension. An almost $\mathrm{CR}$ structure $T_{1,0}(M)$ is integrable, and then $T_{1,0}(M)$ is referred to as a $C R$ structure, if $Z, W \in C^{\infty}\left(U, T_{1,0}(M)\right)$ yields $[Z, W] \in C^{\infty}\left(U, T_{1,0}(M)\right)$ for any open set $U \subset M$. The Levi (or maximally complex) distribution is the real rank $2 n$ distribution on $M$ given by $\mathcal{H} \equiv H(M)=\operatorname{Re}\left\{T_{1,0}(M) \oplus T_{0,1}(M)\right\}$. It carries the complex structure

$$
J: \mathcal{H} \rightarrow \mathcal{H}, \quad J(Z+\bar{Z})=i(Z-\bar{Z}), \quad Z \in T_{1,0}(M) \quad(i=\sqrt{-1}) .
$$

Then $T_{1,0}(M)=\{X-i J X: X \in \mathcal{H}\}$, i.e., $T_{1,0}(M)$ is the eigenbundle of $J$ (the $\mathbb{C}$-linear extension of $J$ to $\mathcal{H} \otimes \mathbb{C}$ ) corresponding to the eigenvalue $i$. The pair $(\mathcal{H}, J)$ (the real manifestation of $T_{1,0}(M)$ ) is often referred to as an almost $\mathrm{CR}$ structure on $M$, as well. A pseudohermitian structure is a differential 1-form $\theta \in \Omega^{1}(M)$ such that $\operatorname{Ker}(\theta)=\mathcal{H}$. Given a pseudohermitian structure $\theta$ on $M$ the Levi form $G_{\theta}$ is given by

$$
G_{\theta}(X, Y)=(d \theta)(X, J Y), \quad X, Y \in \mathcal{H} .
$$

An almost CR structure $(\mathcal{H}, J)$ is nondegenerate if the Levi form $G_{\theta}$ is nondegenerate for some $\theta$. If this is the case $\theta$ is a contact form (i.e., $\theta \wedge(d \theta)^{n}$ is a volume form). An almost CR structure $(\mathcal{H}, J)$ is strictly pseudoconvex if $G_{\theta}$ is positive definite for some $\theta$. Let $(M, \mathcal{H}, J)$ be a nondegenerate almost CR manifold and $\theta$ a fixed contact form on $M$. Let us extend $J$ to an endomorphism $\varphi$ of the tangent bundle by requesting that $\varphi=J$ on $\mathcal{H}$ and $\varphi(T)=0$. Here $T \in \mathfrak{X}(M)$ is unique nowhere zero tangent vector field on $M$ determined by $\theta(T)=1$ and $(d \theta)(T, \cdot)=0$. Then $\varphi^{2}=-I+\theta \otimes T$. The Webster metric is the semi-Riemannian metric $g_{\theta}$ given by

$$
g_{\theta}(X, Y)=(d \theta)(X, J Y), \quad g_{\theta}(X, T)=0, \quad g_{\theta}(T, T)=1
$$


for any $X, Y \in \mathcal{H}$. Then $\left(\varphi, \xi=-T, \eta=-\theta, g=g_{\theta}\right)$ is a contact semiRiemannian structure on $M$. If $G_{\theta}$ is positive definite the Webster metric $g_{\theta}$ is a Riemannian metric (and $(\varphi, \xi, \eta, g)$ is a contact metric structure on $M)$. Conversely any almost contact manifold $(M, \varphi, \xi, \eta)$ carries the almost CR structure given by $\mathcal{H}=\operatorname{Ker}(\eta)$ and $J=\left.\varphi\right|_{\mathcal{H}}$.

\subsection{Levi harmonic maps}

Let $(M, \varphi, \xi, \eta, g)$ be a real $(2 n+1)$-dimensional almost contact semi-Riemannian manifold and $\left(M^{\prime}, g^{\prime}\right)$ a semi-Riemannian manifold. Let $f: M \rightarrow M^{\prime}$ be a $C^{\infty}$ map and $f^{-1} T\left(M^{\prime}\right) \rightarrow M$ the pullback of $T\left(M^{\prime}\right)$ by $f$. Let $\nabla^{\prime f}=f^{-1} \nabla^{\prime}$ be the pullback of the Levi-Civita connection $\nabla^{\prime}$ of $\left(M^{\prime}, g^{\prime}\right)$, i.e., the connection in the vector bundle $f^{-1} T\left(M^{\prime}\right) \rightarrow M$ induced by $\nabla^{\prime}$. If $\left(U, x^{i}\right)$ and $\left(V, y^{\alpha}\right)$ are local coordinate systems on $M$ and $N$ such that $f(U) \subset V$, then $\nabla^{\prime f}$ is locally described by

$$
\nabla_{\partial / \partial x^{j}}^{\prime f}\left(\partial / \partial y^{\beta}\right)^{f}=\frac{\partial f^{\alpha}}{\partial x^{j}}\left(\Gamma_{\alpha \beta}^{\prime \gamma} \circ f\right)\left(\partial / \partial y^{\gamma}\right)^{f},
$$

where $Y^{f}=Y \circ f \in C^{\infty}\left(f^{-1}(V), f^{-1} T\left(M^{\prime}\right)\right)$ denotes the natural lift of $Y \in \mathfrak{X}(V)$ and $\Gamma_{\alpha \beta}^{\prime \gamma}$ are the Christoffel symbols of $\left(M^{\prime}, g^{\prime}\right)$. Let $\mathcal{H}=\operatorname{Ker}(\eta)$ and $J=\left.\varphi\right|_{\mathcal{H}}$ be the almost CR structure underlying $(\varphi, \xi, \eta, g)$. The second fundamental form $\beta_{f}$ of $f$ is given by

$$
\beta_{f}(X, Y)=\nabla_{X}^{\prime f} f_{*} Y-f_{*} \nabla_{X} Y, \quad X, Y \in \mathfrak{X}(M) .
$$

Here $\nabla$ is the Levi-Civita connection of $(M, g)$. Also $f_{*} X \in C^{\infty}\left(f^{-1} T\left(M^{\prime}\right)\right)$ is given by $\left(f_{*} X\right)(x)=\left(d_{x} f\right) X_{x} \in T_{f(x)}\left(M^{\prime}\right)$ for any $x \in M$ and any $X \in \mathfrak{X}(M)$. Next let $\tau_{\mathcal{H}}(f) \in C^{\infty}\left(f^{-1} T\left(M^{\prime}\right)\right)$ be given by

$$
\tau_{\mathcal{H}}(f)=\operatorname{trace}_{g}\left(\Pi_{\mathcal{H}} \beta_{f}\right),
$$

where $\Pi_{\mathcal{H}} \beta_{f}$ is the restriction of $\beta_{f}$ to $\mathcal{H} \otimes \mathcal{H}$.

Definition 2.1. A $C^{\infty}$ map $f: M \rightarrow M^{\prime}$ is Levi harmonic with respect to $\mathcal{H}=\operatorname{Ker}(\eta)$ if $\tau_{\mathcal{H}}(f)=0$.

Next, we recall the following:

Definition 2.2. A $C^{\infty}$ map $f: M \rightarrow M^{\prime}$ of almost $\mathrm{CR}$ manifolds is a $C R$ map if

$$
\left(d_{x} f\right) \mathcal{H}_{x} \subset \mathcal{H}\left(M^{\prime}\right)_{f(x)}, \quad\left(d_{x} f\right) \circ J_{x}=J_{f(x)}^{\prime} \circ\left(d_{x} f\right)
$$

for any $x \in M$.

Typical examples of CR maps are got as traces of holomorphic maps of Kaehlerian manifolds on real hypersurfaces. Precisely let $\bar{M}$ be a Kaehlerian manifold. Any orientable real hypersurface $M \subset \bar{M}$ admits a natural almost contact metric structure (cf. e.g. [5]). If $M^{\prime} \subset \bar{M}^{\prime}$ is another oriented real hypersurface in the Kaehlerian manifold $\bar{M}^{\prime}$ and $F: \bar{M} \rightarrow \bar{M}^{\prime}$ is a holomorphic map such that $F(M) \subset M^{\prime}$, then $\left.f \equiv F\right|_{M}: M \rightarrow M^{\prime}$ is a CR map. It 
should be emphasized that, in spite of our metric approach [where the wealth of additional first order geometric structure $(\varphi, \xi, \eta, g)$ is meant to "compensate" for the lack of integrability of $\left.T_{1,0}(M)\right]$ the property $(2.7)$ is tied to the almost CR structures alone. In particular the statements above hold true for traces of holomorphic maps among indefinite Kaehlerian manifolds (cf. E. Barros and A. Romero, [3], for definitions and examples). Indeed let $\bar{M}$ be an indefinite Kaehlerian manifold and $M \subset \bar{M}$ an orientable real hypersurface. The indefinite Kaehler structure of $\bar{M}$ induces on $M$ an almost contact semi-Riemannian structure (cf. A. Bejancu and K. L. Duggal, [4]).

Let $\theta$ and $\theta^{\prime}$ be pseudohermitian structures on the almost CR manifolds $M$ and $M^{\prime}$, respectively. If $f: M \rightarrow M^{\prime}$ is a CR map, then

$$
f^{*} \theta^{\prime}=\mu \theta
$$

for some $\mu \in C^{\infty}(M)$.

Definition 2.3. A CR map $f$ is pseudohermitian if $\mu=c$ for some $c \in \mathbb{R}$. Also $f$ is isopseudohermitian if $c=1$.

\section{The $\varphi$-condition and the condition $(A)$}

In [10] we adopted the following:

Definition 3.1. We say that an almost contact semi-Riemannian manifold $(M, \varphi, \xi, \eta, g)$ satisfies the $\varphi$-condition if

$$
\nabla_{\varphi X} \varphi X+\nabla_{X} X=\varphi[\varphi X, X]
$$

for any $X \in \mathcal{H}$.

Now, we consider the following tensor

$$
\mathcal{P}(X, Y)=\left(\nabla_{X} \varphi\right) \varphi Y-\left(\nabla_{\varphi X} \varphi\right) Y
$$

for any $X, Y \in \mathcal{H}=\operatorname{ker}(\eta) . \mathcal{P}$ is a tensor of type $(1,2)$ which is $\varphi$-invariant, i.e., $\mathcal{P}(\varphi X, \varphi Y)=\mathcal{P}(X, Y)$, moreover we note that $M$ satisfies the $\varphi$-condition if and only if the tensor $\mathcal{P}$ is skew-symmetric, that is $\mathcal{P}(X, X)=0$. Next, we put

and

$$
\mathcal{H}^{+}=T_{1,0}(M)=\{X-i \varphi X, X \in \mathcal{H}\}
$$

$$
\mathcal{H}^{-}=T_{0,1}(M)=\{X+i \varphi X, X \in \mathcal{H}\} .
$$

Then the complexified tangent bundle of $M$ splits into the direct sum of the tangent bundles $\mathcal{H}^{+}, \mathcal{H}^{-}$and $\mathbb{C} \xi$. Besides $\mathcal{H}^{+}$(resp. $\mathcal{H}^{-}$) is the eigenbundle of $\varphi$ (the $\mathbb{C}$-linear extension to $\mathcal{H} \otimes \mathbb{C}$ ) corresponding to the eigenvalue $i$ (resp. $-i)$.

We note that the tensor field $\varphi$ of an almost contact semi-Riemannian manifold defines a $f$-structure, that is,

$$
\varphi^{3}+\varphi=0 .
$$


Then, following Rawnsley [17] (cf. p. 91), we say that the almost contact semiRiemannian structure defines the so-called condition $(A)$, if

$$
\nabla_{V} W \in \mathcal{H}^{+} \quad \text { for any } \quad V \in \mathcal{H}^{-} \text {and } W \in \mathcal{H}^{+} .
$$

Rawnsley introduced a such condition in order to study the harmonicity of $f$-holomorphic maps between an almost Hermitian manifold with coclosed Kaehler form and a Riemannian manifold equipped with a $f$-structure which satisfies condition $(A)$.

Since $\left(\nabla_{V} \varphi\right) W=\nabla_{V} \varphi W-\varphi \nabla_{V} W$, condition $(A)$ is equivalent to (cf. also [1] Proposition 2.6)

$$
\left(\nabla_{V} \varphi\right) W=0 \quad \text { for any } \quad V \in \mathcal{H}^{-} \text {and } W \in \mathcal{H}^{+} .
$$

An almost Hermitian structure satisfying the condition $(A)$ is said to be a $(1,2)$-symplectic structure (see also [1] p. 251).

We remark that the condition $(A)$, that is $(3.2)$, is satisfied if and only if

$$
\left(\nabla_{X} \varphi\right) Y+\left(\nabla_{\varphi X} \varphi\right) \varphi Y=0, \quad \text { equivalently } \mathcal{P}(X, Y)=0
$$

for any $X, Y \in \mathcal{H}$. Moreover, the condition (3.3) implies the $\varphi$-condition, that is $\mathcal{P}(X, X)=0$. More precisely, one gets

$$
\mathcal{P}(X, Y)=0 \Longleftrightarrow \mathcal{P}(X, X)=0 \quad \text { and } \quad \mathcal{P}(X, \varphi X)=0 .
$$

Therefore, the condition $(A)$ implies the $\varphi$-condition. On the other hand, the converse does not hold. In fact, the condition $\mathcal{P}(X, \varphi X)=0$ means that

$$
[X, \varphi X]=\varphi\left(\nabla_{\varphi X} \varphi X+\nabla_{X} X\right) \in \mathcal{H}
$$

for any $X \in \mathcal{H}$. But, if $M$ is a contact semi-Riemannian manifold

$$
\begin{aligned}
\varepsilon g([X, \varphi X], \xi) & =\eta([X, \varphi X])=-2(\mathrm{~d} \eta)(\mathrm{X}, \varphi \mathrm{X}) \\
& =-2 g\left(X, \varphi^{2} X\right)=2 g(X, X) \neq 0
\end{aligned}
$$

for $X \in \mathcal{H}, X \neq 0, X$ spacelike or timelike.

So contact semi-Riemannian structures do not satisfy the condition $(A)$. However contact semi-Riemannian structures satisfy the $\varphi$-condition. In fact, by (2.4), for any $X, Y \in \mathcal{H}$

$$
\nabla_{X} \varphi Y-\varphi \nabla_{X} Y-\nabla_{\varphi X} Y-\varphi \nabla_{\varphi X} \varphi Y=2 g(X, Y) \xi
$$

In particular for $Y=\varphi X$ one derives (3.1). Hence any contact semi-Riemannian manifold satisfies the $\varphi$-condition. Therefore, we get the following:

Theorem 3.2. Let $(M, \varphi, \xi, \eta, g)$ be an almost contact semi-Riemannian manifold. If the almost contact semi-Riemannian structure satisfies the condition $(A)$, then it satisfies the $\varphi$-condition. The converse does not hold.

So, our $\varphi$-condition extends the condition $(A)$ of Rawnsley [17]. 
Remark 3.3. As emphasized in [10] the class of almost contact semi-Riemannian manifolds obeying to (3.1) is quite large. For instance, contact semi-Riemannian manifolds, orientable real hypersurfaces in an indefinite Kaehler manifold (with the induced almost contact semi-Riemannian structure) and quasi-cosimplectic manifolds (cf. Section 6) satisfy the $\varphi$-condition.

\section{Harmonicity between almost contact semi-Riemannian manifolds}

In this section we establish the following theorem which extends Theorem 3.9 of [10].

Theorem 4.1. Let $(M, \varphi, \xi, \eta, g)$ and $\left(M^{\prime}, \varphi^{\prime}, \xi^{\prime}, \eta^{\prime}, g^{\prime}\right)$ be two almost contact semi-Riemannian manifolds with $\operatorname{dim}(M)=2 n+1$. Then, for each CR map $f: M \rightarrow M^{\prime}$

$$
\begin{aligned}
\tau_{\mathcal{H}}(f)= & -\operatorname{trace}(\varphi \nabla \xi) \varphi^{\prime f} f_{*} \xi+\varphi^{\prime f}\left(\operatorname{trace}_{\mid \mathcal{H}} f^{*} \nabla^{\prime} \varphi^{\prime}\right) \\
& -\left(\operatorname{trace}_{\mid \mathcal{H}} f^{*} \nabla^{\prime} \eta^{\prime}\right) \xi^{\prime}+f_{*}\left(\varphi \nabla^{*} \varphi+(\operatorname{div} \xi) \xi+\varepsilon \nabla_{\xi} \xi\right),
\end{aligned}
$$

where $\varepsilon=g(\xi, \xi), \varphi^{\prime f}: f^{-1} T\left(M^{\prime}\right) \rightarrow f^{-1} T\left(M^{\prime}\right)$ is the pullback of $\varphi^{\prime}$ by $f$, and $\nabla^{*}$ is the operator formal adjoint of $\nabla$.

Proof. The tangent bundle to any $(2 n+1)$-dimensional almost contact semiRiemannian manifold $M$ admits a local semi-orthonormal frame (a $\varphi$-basis), i.e., a frame of the form $\left\{\xi, E_{\alpha}, \varphi E_{\alpha}: 1 \leq \alpha \leq n\right\}$. By (2.1) if $E_{\alpha}$ is a spacelike (respectively timelike), then $\varphi E_{\alpha}$ is spacelike (respectively timelike). In particular a semi-Riemannian metric compatible with an almost contact structure has either signature $(2 p+1,2 n-2 p)$ or signature $(2 p, 2 n-2 p+1)$, according to whether $\xi$ is spacelike or timelike.

Let $\left\{\xi, E_{\alpha}, \varphi E_{\alpha}: 1 \leq \alpha \leq n\right\}$ be a $\varphi$-basis and let us set $\varepsilon_{\alpha}=g\left(E_{\alpha}, E_{\alpha}\right) \in$ $\{ \pm 1\}$. Then one has

$$
\tau_{\mathcal{H}}(f)=\sum_{\alpha=1}^{n} \varepsilon_{\alpha}\left\{\nabla_{E_{\alpha}}^{f} f_{*} E_{\alpha}-f_{*} \nabla_{E_{\alpha}} E_{\alpha}+\nabla_{\varphi E_{\alpha}}^{\prime f} f_{*} \varphi E_{\alpha}-f_{*} \nabla_{\varphi E_{\alpha}} \varphi E_{\alpha}\right\}
$$

We consider the operator $\nabla^{*}$, the formal adjoint of $\nabla$ (see for example [9], pp. 108-110), thus if $S$ is a tensor of type $(1,1), \nabla^{*} S=-\operatorname{trace} \nabla S$. Then,

$$
\begin{aligned}
-\nabla^{*} \varphi & \left.=\sum_{\alpha=1}^{n} \varepsilon_{\alpha}\left\{\left(\nabla_{E_{\alpha}} \varphi\right) E_{\alpha}+\left(\nabla_{\varphi E_{\alpha}} \varphi\right) \varphi E_{\alpha}\right)\right\}+\varepsilon\left(\nabla_{\xi} \varphi\right) \xi \\
& =\sum_{\alpha=1}^{n} \varepsilon_{\alpha}\left\{\left[E_{\alpha}, \varphi E_{\alpha}\right]-\varphi\left(\nabla_{E_{\alpha}} E_{\alpha}+\nabla_{\varphi E_{\alpha}} \varphi E_{\alpha}\right)\right\}-\varepsilon \varphi \nabla_{\xi} \xi,
\end{aligned}
$$

and hence

$$
-\varphi \nabla^{*} \varphi=\sum_{\alpha=1}^{n} \varepsilon_{\alpha}\left\{\varphi\left[E_{\alpha}, \varphi E_{\alpha}\right]-\varphi^{2}\left(\nabla_{E_{\alpha}} E_{\alpha}+\nabla_{\varphi E_{\alpha}} \varphi E_{\alpha}\right)\right\}+\varepsilon \nabla_{\xi} \xi
$$




$$
=\sum_{\alpha=1}^{n} \varepsilon_{\alpha}\left\{\left(\nabla_{E_{\alpha}} E_{\alpha}+\nabla_{\varphi E_{\alpha}} \varphi E_{\alpha}\right)-\varphi\left[\varphi E_{\alpha}, E_{\alpha}\right]\right\}+(\operatorname{div} \xi) \xi+\varepsilon \nabla_{\xi} \xi
$$

that is,

$$
\begin{aligned}
& \sum_{\alpha=1}^{n} \varepsilon_{\alpha}\left(\nabla_{E_{\alpha}} E_{\alpha}+\nabla_{\varphi E_{\alpha}} \varphi E_{\alpha}\right) \\
= & \sum_{\alpha=1}^{n} \varepsilon_{\alpha} \varphi\left[\varphi E_{\alpha}, E_{\alpha}\right]-\varphi \nabla^{*} \varphi-(\operatorname{div} \xi) \xi-\varepsilon \nabla_{\xi} \xi .
\end{aligned}
$$

Moreover,

$$
\begin{aligned}
& \varphi^{\prime f}\left(\operatorname{trace}_{\mid \mathcal{H}} f^{*} \nabla^{\prime} \varphi^{\prime}\right) \\
= & \varphi^{\prime f} \sum_{\alpha=1}^{n} \varepsilon_{\alpha}\left\{\left(\nabla_{f_{*} E_{\alpha}}^{\prime} \varphi^{\prime}\right) f_{*} E_{\alpha}+\left(\nabla_{f_{*} \varphi E_{\alpha}}^{\prime} \varphi^{\prime}\right) f_{*} \varphi E_{\alpha}\right\} \\
= & \sum_{\alpha=1}^{n} \varepsilon_{\alpha}\left\{\varphi^{\prime f}\left[f_{*} E_{\alpha}, \varphi^{\prime f} f_{*} E_{\alpha}\right]-\left(\varphi^{\prime f}\right)^{2}\left(\nabla_{f_{*} E_{\alpha}}^{\prime} f_{*} E_{\alpha}+\nabla_{f_{*} \varphi E_{\alpha}}^{\prime} f_{*} \varphi E_{\alpha}\right)\right\} \\
= & \sum_{\alpha=1}^{n} \varepsilon_{\alpha}\left\{\varphi^{\prime f}\left[f_{*} E_{\alpha}, \varphi^{\prime f} f_{*} E_{\alpha}\right]+\nabla_{f_{*} E_{\alpha}}^{\prime} f_{*} E_{\alpha}\right. \\
& \left.+\nabla_{f_{*} \varphi E_{\alpha}}^{\prime} f_{*} \varphi E_{\alpha}-\eta^{\prime}\left(\nabla_{f_{*} E_{\alpha}}^{\prime} f_{*} E_{\alpha}+\nabla_{f_{*} \varphi E_{\alpha}}^{\prime} f_{*} \varphi E_{\alpha}\right) \xi^{\prime}\right\},
\end{aligned}
$$

and (as $f$ is a CR map)

$$
\begin{aligned}
\left(\operatorname{trace}_{\mid \mathcal{H}} f^{*} \nabla^{\prime} \eta^{\prime}\right) & \left.=\sum_{\alpha=1}^{n} \varepsilon_{\alpha}\left\{\left(\nabla_{f_{*} E_{\alpha}}^{\prime} \eta^{\prime}\right) f_{*} E_{\alpha}+\left(\nabla_{f_{*} \varphi E_{\alpha}}^{\prime} \eta^{\prime}\right) f_{*} \varphi E_{\alpha}\right)\right\} \\
& =-\sum_{\alpha=1}^{n} \varepsilon_{\alpha} \eta^{\prime}\left(\nabla_{f_{*} E_{\alpha}}^{\prime} f_{*} E_{\alpha}+\nabla_{f_{*} \varphi E_{\alpha}}^{\prime} f_{*} \varphi E_{\alpha}\right)
\end{aligned}
$$

give

$$
\text { (4.4) } \begin{aligned}
& \sum_{\alpha=1}^{n} \varepsilon_{\alpha}\left\{\left(\nabla_{f_{*} E_{\alpha}}^{\prime} f_{*} E_{\alpha}+\nabla_{f_{*} \varphi E_{\alpha}}^{\prime} f_{*} \varphi E_{\alpha}\right)\right. \\
= & \varphi^{\prime f}\left(\operatorname{trace}_{\mid \mathcal{H}} f^{*} \nabla^{\prime} \varphi^{\prime}\right)-\left(\operatorname{trace}_{\mid \mathcal{H}} f^{*} \nabla^{\prime} \eta^{\prime}\right) \xi^{\prime}+\sum_{\alpha=1}^{n} \varepsilon_{\alpha}\left\{\varphi^{\prime f}\left[f_{*} \varphi E_{\alpha}, f_{*} E_{\alpha}\right]\right\} .
\end{aligned}
$$

Then, (4.2), (4.3) and (4.4) imply

(4.5) $\tau_{\mathcal{H}}(f)=\sum_{\alpha=1}^{n} \varepsilon_{\alpha}\left\{\left(\varphi^{\prime f} f_{*}-f_{*} \varphi\right)\left[\varphi E_{\alpha}, E_{\alpha}\right]\right\}+f_{*}\left(\varphi \nabla^{*} \varphi+(\operatorname{div} \xi) \xi+\varepsilon \nabla_{\xi} \xi\right)$

$$
+\varphi^{\prime f}\left(\operatorname{trace}_{\mid \mathcal{H}} f^{*} \nabla^{\prime} \varphi^{\prime}\right)-\left(\operatorname{trace}_{\mid \mathcal{H}} f^{*} \nabla^{\prime} \eta^{\prime}\right) \xi^{\prime} .
$$

Note that $\varphi^{\prime f} f_{*}-f_{*} \varphi=0$ on $\mathcal{H}$,

$$
\left[\varphi E_{\alpha}, E_{\alpha}\right]=-\varphi^{2}\left[\varphi E_{\alpha}, E_{\alpha}\right]+g\left(\left[\varphi E_{\alpha}, E_{\alpha}\right], \xi\right) \xi
$$


and

$$
\begin{aligned}
\sum_{\alpha=1}^{n} \varepsilon_{\alpha} g\left(\left[\varphi E_{\alpha}, E_{\alpha}\right], \xi\right) & =\sum_{\alpha=1}^{n} \varepsilon_{\alpha}\left\{g\left(\nabla_{\varphi E_{\alpha}} E_{\alpha}, \xi\right)-g\left(\nabla_{E_{\alpha}} \varphi E_{\alpha}, \xi\right)\right\} \\
& =\sum_{\alpha=1}^{n} \varepsilon_{\alpha}\left\{-g\left(\nabla_{\varphi E_{\alpha}} \xi, E_{\alpha}\right)+g\left(\nabla_{E_{\alpha}} \xi, \varphi E_{\alpha}\right)\right\} \\
& =-\sum_{\alpha=1}^{n} \varepsilon_{\alpha}\left\{g\left(\varphi \nabla_{\varphi E_{\alpha}} \xi, \varphi E_{\alpha}\right)+g\left(\varphi \nabla_{E_{\alpha}} \xi, E_{\alpha}\right)\right\} \\
& =-\operatorname{trace}(\varphi \nabla \xi)+\varepsilon g\left(\varphi \nabla_{\xi} \xi, \xi\right)=-\operatorname{trace}(\varphi \nabla \xi)
\end{aligned}
$$

So, from (4.5) we get (4.1).

Next, if $M$ and $M^{\prime}$ satisfy the $\varphi$-condition, from (4.3) and (4.4) we have

$$
\varphi \nabla^{*} \varphi+(\operatorname{div} \xi) \xi+\varepsilon \nabla_{\xi} \xi=0
$$

and

$$
\varphi^{\prime f}\left(\operatorname{trace}_{\mathcal{H}} f^{*} \nabla^{\prime} \varphi^{\prime}\right)-\left(\operatorname{trace}_{\mid \mathcal{H}} f^{*} \nabla^{\prime} \eta^{\prime}\right) \xi^{\prime}=0 .
$$

Therefore we obtain:

Corollary $4.2([10])$. Let $(M, \varphi, \xi, \eta, g)$ and $\left(M^{\prime}, \varphi^{\prime}, \xi^{\prime}, \eta^{\prime}, g^{\prime}\right)$ be two almost contact semi-Riemannian manifolds with $\operatorname{dim}(M)=2 n+1$, satisfying the $\varphi$ condition. Then, for any $C R$ map $f: M \rightarrow M^{\prime}$

$$
\tau_{\mathcal{H}}(f)=-\operatorname{trace}(\varphi \nabla \xi) \varphi^{\prime f} f_{*} \xi .
$$

If additionally $(M, \varphi, \xi, \eta, g)$ is a contact semi-Riemannian manifold, then

$$
\tau_{\mathcal{H}}(f)=-2 n \varepsilon \varphi^{\prime f} f_{*} \xi
$$

where $\varepsilon=g(\xi, \xi)$. Hence $f$ is Levi harmonic if and only if $f_{*} \xi$ is collinear to $\xi^{\prime}$.

Moreover, since

$$
\tau(f)=\tau_{\mathcal{H}}(f)+\varepsilon\left(\nabla_{f_{*} \xi}^{\prime} f_{*} \xi-f_{*} \nabla_{\xi} \xi\right),
$$

from Theorem 4.1, we get:

Theorem 4.3. Let $(M, \varphi, \xi, \eta, g)$ and $\left(M^{\prime}, \varphi^{\prime}, \xi^{\prime}, \eta^{\prime}, g^{\prime}\right)$ be two almost contact semi Riemannian manifolds satisfying the $\varphi$-condition, with $\xi$ geodesic and $\operatorname{trace}(\varphi \nabla \xi)=0$. Then, a $C R$ map $f: M \rightarrow M^{\prime}$ is a harmonic map if and only if $f_{*} \xi$ is a geodesic vector field.

An application of this theorem is given in Section 6 . 


\section{Levi pluriharmonicity}

Let $(M, g, J)$ be a Kaehler manifold and $\left(M^{\prime}, g^{\prime}\right)$ a Riemannian manifold. Following [12] a $C^{\infty}$ map $f: M \rightarrow M^{\prime}$ is said to be pluriharmonic if the second fundamental form $\beta_{f}$ satisfies

$$
\beta_{f}(J X, J Y)+\beta_{f}(X, Y)=0, \quad \forall X, Y \in \mathfrak{X}(M) .
$$

Then, we introduce the following:

Definition 5.1. Let $(M, \varphi, \xi, \eta, g)$ be an almost contact semi-Riemannian manifold and $\left(M,^{\prime}, g^{\prime}\right)$ a semi-Riemannian manifold. A $C^{\infty}$ map $f: M \rightarrow M^{\prime}$ is said to be Levi pluriharmonic if the second fundamental form $\beta_{f}$ satisfies

$$
\beta_{f}(\varphi X, \varphi Y)+\beta_{f}(X, Y)=0, \quad \forall X, Y \in \mathcal{H},
$$

equivalently

$$
\beta_{f}(\varphi X, \varphi X)+\beta_{f}(X, X)=0, \quad \forall X \in \mathcal{H} .
$$

Of course Levi pluriharmonicity implies Levi harmonicity. Now, we show the following.

Theorem 5.2. Let $(M, \varphi, \xi, \eta, g)$ be a contact semi-Riemannian manifold and $\left(M^{\prime}, \varphi^{\prime}, \xi^{\prime}, \eta^{\prime}, g^{\prime}\right)$ an almost contact semi-Riemannian manifold satisfying the $\varphi$-condition with $\xi^{\prime}$ geodesic vector field. If $f: M \rightarrow M^{\prime}$ is a $C R$ map, then $f$ is Levi pluriharmonic if and only if $f$ is a pseudohermitian map.

Proof. Necessity. We suppose that $f$ is a pseudohermitian map. Thus $f$ is a CR map and $f_{*} \xi=c \xi^{\prime}$ with $c \in \mathbb{R}$. This implies that

$$
f_{*} \varphi X=\varphi^{\prime f} f_{*} X, \quad \forall X \in \mathfrak{X}(M) .
$$

Then, as $f$ is a CR map one has

$$
\begin{aligned}
\beta_{f}(\varphi X, \varphi X)+\beta_{f}(X, X) & =\left\{\nabla_{X}^{\prime f} f_{*} X-f_{*} \nabla_{X} X+\nabla_{\varphi X}^{\prime f} f_{*} \varphi-f_{*} \nabla_{\varphi X} \varphi X\right\} \\
& =\left\{\nabla_{X}^{\prime f} f_{*} X+\nabla_{\varphi X}^{\prime f} \varphi^{\prime} f_{*} X-f_{*}\left(\nabla_{X} X+\nabla_{\varphi X} \varphi X\right)\right\} .
\end{aligned}
$$

Next, since both $M$ and $M^{\prime}$ satisfy the $\varphi$-condition, by (5.2)

$$
\beta_{f}(\varphi X, \varphi X)+\beta_{f}(X, X)=\left(\varphi^{\prime f} f_{*}-f_{*} \varphi\right)[\varphi X, X]=0 .
$$

Hence $f$ is Levi pluriharmonic. Conversely, we suppose that $f$ is Levi pluriharmonic. Then $f$ is Levi harmonic. On the other hand by Corollary 4.2

$$
\tau_{\mathcal{H}}(f)=-\operatorname{trace}(\varphi \nabla \xi) \varphi^{\prime f} f_{*} \xi .
$$

Since for a contact semi-Riemannian manifold trace $(\varphi \nabla \xi)=2 n \varepsilon \neq 0$, we get $\varphi^{\prime} f_{*} \xi=0$ and thus $f_{*} \xi=\lambda \xi^{\prime}$ for some $\lambda \in C^{\infty}(M, \mathbb{R})$, that is $f^{*} \eta^{\prime}=\lambda \eta$. Then

$$
\begin{aligned}
\xi(\lambda) \eta-d \lambda & =(d \lambda \wedge \eta)(\xi, \cdot)=d(\lambda \eta)(\xi, \cdot) \\
& =\left(d f^{*} \eta^{\prime}\right)(\xi, \cdot)=\left(f^{*} d \eta^{\prime}\right)(\xi, \cdot) \\
& =\left(d \eta^{\prime}\right)\left(f_{*} \xi, f_{*} \cdot\right)=\lambda\left(d \eta^{\prime}\right)\left(\xi^{\prime}, f_{*} \cdot\right) .
\end{aligned}
$$


On the other hand, since $\xi^{\prime}$ is a geodesic vector field, we have

$$
\begin{aligned}
2\left(d \eta^{\prime}\right)\left(\xi^{\prime}, X\right) & =\xi^{\prime} \eta^{\prime}(X)-X \eta^{\prime}\left(\xi^{\prime}\right)-\eta^{\prime}\left(\left[\xi^{\prime}, X\right]\right) \\
& =\varepsilon g^{\prime}\left(\nabla_{\xi^{\prime}}^{\prime} \xi^{\prime}, X\right) \\
& =0 .
\end{aligned}
$$

Therefore $d \lambda=\xi(\lambda) \eta$ and consequently

$$
\xi(\lambda) \eta \wedge d \eta=d \lambda \wedge d \eta=-d(d \lambda \wedge \eta)=-d(\xi(\lambda) \eta \wedge \eta)=0 .
$$

So, since $\eta$ is a contact form, we get that $\xi(\lambda)=0$. This gives that $\lambda$ is a constant and $f$ is a pseudohermitian map.

Let $(\bar{M}, \bar{\varphi}, \bar{\xi}, \bar{\eta})$ be an almost contact manifold. A submanifold $M$ of $\bar{M}$ is called an invariant submanifold if $\bar{\varphi}_{p} T_{p}(M) \subset T_{p}(M)$ for any $p \in M$. Then, we have two cases (cf. [18]): I) $\bar{\xi}$ is tangent to $M$ (and then $M$ is odd-dimensional) or II) $\bar{\xi}$ is transverse to $M$ (and then $M$ is even-dimensional). When $\bar{M}$ is a contact semi-Riemannian manifold case II doesn't occur (cf. [5], p. 122). We consider the case I, and then $M$ carries the induced almost contact structure $(\varphi, \xi, \eta)$ given by

$$
\bar{\varphi} \circ i_{*}=i_{*} \circ \varphi, \quad \eta=i^{*} \bar{\eta}, \quad \bar{\xi}=i_{*} \xi
$$

where $i: M \rightarrow \bar{M}$ is the inclusion. In particular $i$ is a CR map.

Now, we assume that $(\bar{M}, \bar{\varphi}, \bar{\xi}, \bar{\eta}, \bar{g})$ is an almost contact semi-Riemannian manifold, $M$ an odd-dimensional invariant submanifold of $\bar{M}$ equipped with the induced almost contact structure $(\varphi, \xi, \eta)$, and let $g$ be a semi-Riemannian metric on $M$ such that $g=i^{*} \bar{g}$. Then $g$ is compatible with $(\varphi, \xi, \eta)$, that is $(\varphi, \xi, \eta, g)$ is an almost contact semi-Riemannian structure on $M$. In such case we say that $M$ is an invariant semi-Riemannian submanifold of $\bar{M}$.

Suppose that $\bar{M}$ satisfies the $\bar{\varphi}$-condition. Then for any $X \in \mathcal{H}$

$$
\bar{\nabla}_{\bar{\varphi} X} \bar{\varphi} X+\bar{\nabla}_{X} X=\bar{\varphi}[\bar{\varphi} X, X],
$$

and hence (by the Gauss formula)

$$
\nabla_{\varphi X} \varphi X+\alpha(\varphi X, \varphi X)+\nabla_{X} X+\alpha(X, X)=\varphi[\varphi X, X] .
$$

The tangential and normal components of (5.4) give

$$
\nabla_{\varphi X} \varphi X+\nabla_{X} X=\varphi[\varphi X, X]
$$

and

$$
\alpha(\varphi X, \varphi X)+\alpha(X, X)=0
$$

for any $X \in \mathcal{H}$. Thus by (5.5) the inclusion $i$ is Levi-pluriharmonic.

Next, we note that if $(\bar{\varphi}, \bar{\xi}, \bar{\eta}, \bar{g})$ is a contact semi-Riemannian structure on $\bar{M}$, then from (5.3) follows that

$$
\begin{aligned}
d \eta & =d i^{*} \bar{\eta}=i^{*} d \bar{\eta}=\bar{g}\left(i_{*}, \bar{\varphi} \circ i_{*}\right) \\
& =\bar{g}\left(i_{*}, i_{*} \circ \varphi\right)=\left(i^{*} \bar{g}\right)(\cdot, \varphi)=g(\cdot, \varphi),
\end{aligned}
$$


that is, $(M, \varphi, \xi, \eta, g)$ is an invariant contact semi-Riemannian manifold of $\bar{M}$.

Therefore, using also Theorem 5.2, we get:

Theorem 5.3. Let $(\bar{M}, \bar{\varphi}, \bar{\xi}, \bar{\eta}, \bar{g})$ be an almost contact semi-Riemannian manifold satisfying the $\bar{\varphi}$-condition. If $(M, g)$ is an odd-dimensional invariant semiRiemannian submanifold of $\bar{M}$, then the inclusion $i: M \rightarrow \bar{M}$ is Levi pluriharmonic. In particular, if $\bar{M}$ is a contact semi-Riemannian manifold and $M$ is an invariant semi-Riemannian submanifold of $\bar{M}$, then the inclusion $i: M \rightarrow \bar{M}$ is Levi pluriharmonic and a pseudohermitian map.

Now we give some examples of Levi pluriharmonic maps.

Sasakian space forms. Let $M^{2 n+3}(c)$ be a complete simply connected Sasakian manifold of constant $\phi$-sectional curvature $c$. As well known $M^{2 n+3}(c)$ is equivalent to one of the Sasakian manifolds $S^{2 n+3}, \mathbb{R}^{2 n+3}$ or $D^{n+1} \times \mathbb{R}$ equipped with Sasakian structures of $\varphi$-sectional curvature $c>-3, c=-3$ and $c<-3$, respectively, where $D^{n+1} \subset \mathbb{C}^{n+1}$ is a simply connected bounded domain. Then $M^{2 n+1}(c)$ is an invariant submanifold of $M^{2 n+3}(c)$ (cf. [19], p. 328) and, by Theorem 5.3, the inclusion $i: M^{2 n+1}(c) \rightarrow M^{2 n+3}(c)$ is Levi pluriharmonic and a pseudohermitian map.

The Brieskorn sphere. Let $\mathbb{C}^{n+1}$ with the Cartesian complex coordinates $z=\left(z_{0}, \ldots, z_{n}\right)$ and $a=\left(a_{0}, \ldots, a_{n}\right) \in \mathbb{Z}^{n+1}$ such that $a_{j} \geq 2$. Let us consider the polynomial $P_{a}(z)=\sum_{j=0}^{n} z_{j}^{a_{j}} \in \mathbb{C}[z]$. Then $B^{2 n}(a)=\left\{z \in \mathbb{C}^{n+1}\right.$ : $\left.P_{a}(z)=0\right\}$ is an algebraic hypersurface in $\mathbb{C}^{n+1}$ and $B^{2 n}(a) \backslash\{0\}$ is an $n$ dimensional complex submanifold. Let us set $\Sigma^{2 n-1}(a)=B^{2 n}(a) \cap S^{2 n+1}$ (the Brieskorn sphere determined by $a$ ). By a result in [19], pp. 303-305, $S^{2 n+1}$ admits a Sasakian structure $(\bar{\varphi}, \bar{\xi}, \bar{\eta}, \bar{g}$ ) (distinct from the standard Sasakian structure) such that $\Sigma^{2 n-1}(2, \ldots, 2)$ is an invariant submanifold of $\left(S^{2 n+1}, \bar{\varphi}, \bar{\xi}, \bar{\eta}, \bar{g}\right)$. Thus, the inclusion $i: \Sigma^{2 n-1}(2, \ldots, 2) \rightarrow S^{2 n+1}$ is Levi pluriharmonic and a pseudohermitian map.

Let $Q^{n}=\pi_{0}\left(B^{2 n+2}(2, \ldots, 2)\right)$ be the complex quadric, where $\pi_{0}: \mathbb{C}^{n+2} \backslash$ $\{0\} \rightarrow \mathbb{C} P^{n+1}$ is the projection. Let $\pi: S^{2 n+3} \rightarrow \mathbb{C} P^{n+1}$ be the Hopf fibration. The saturated set $P=\pi^{-1}\left(Q^{n}\right)$ is the total space a circle bundle $S^{1} \rightarrow P \rightarrow$ $Q^{n}$. Then $P$ is a invariant submanifold of the sphere $S^{2 n+3}$ equipped with the standard Sasakian structure (cf. [19], p. 328). Hence the inclusion $P \rightarrow S^{2 n+3}$ is Levi pluriharmonic and a pseudohermitian map.

Remark 5.4. Let $(\bar{M}, \bar{\varphi}, \bar{\xi}, \bar{\eta}, \bar{g})$ be a contact Riemannian manifold and $M$ an invariant submanifold of $\bar{M}$ equipped with the induced contact Riemannian structure $(M, \varphi, \xi, \eta, g)$. We put

$$
\bar{g}_{L}=\bar{g}-2 \bar{\eta} \otimes \bar{\eta} \quad \text { and } \quad g_{L}=g-2 \eta \otimes \eta=i^{*} \bar{g}_{L} .
$$

Then, $\left(\bar{\varphi}, \bar{\xi}, \bar{\eta}, \bar{g}_{L}\right)$ is a contact Lorentzian structure on $\bar{M}$ with $\bar{\xi}$ time-like, and such structure is Sasakian if and only if the corresponding Riemannian structure is Sasakian [6]. Moreover, $M$ equipped with the contact Lorentzian structure $\left(\varphi, \xi, \eta, g_{L}\right)$ is an invariant Lorentzian submanifold of the contact Lorentzian 
manifold $\left(\bar{M}, \bar{\varphi}, \bar{\xi}, \bar{\eta}, \bar{g}_{L}\right)$. Therefore, the inclusion $i: M \rightarrow \bar{M}$ is Levi pluriharmonic and a pseudohermitian map between two contact Lorentzian manifolds. In particular, the above examples define Levi pluriharmonic and pseudohermitian maps between contact Lorentz-Sasakian manifolds.

\section{Levi harmonicity of quasi-cosymplectic manifolds}

In this section we study the harmonicity of a CR map defined on a quasicosymplectic manifold. Recall that an almost contact Riemannian manifold $M$ is said to be quasi-cosymplectic (cf. [7], and [8] p. 666) if

$$
\left(\nabla_{X} \varphi\right) Y+\left(\nabla_{\varphi X} \varphi\right) \varphi Y=\eta(Y) \nabla_{\varphi X} \xi, \quad X, Y \in \mathfrak{X}(M) .
$$

By a result of Z. Olszak (cf. [13], Lemma 2.2, p. 240) any almost cosymplectic manifold satisfies (6.6). So, the class of quasi-cosymplectic manifolds is large, it contains the classes of cosymplectic and almost cosymplectic manifolds. In the paper [14] we classified all simply connected homogeneous almost cosymplectic three-manifolds. Moreover, there exist examples of quasi-cosymplectic manifolds which are not almost cosymplectic (see, for example [7], and [8] p. 668).

Next, let $M$ be a quasi-cosymplectic manifold. Then, for $X \in \operatorname{ker}(\eta)$ and $Y=\varphi X$, (6.6) implies

$$
\left(\nabla_{X} \varphi\right) \varphi X=\left(\nabla_{\varphi X} \varphi\right) X
$$

equivalently $\nabla_{X} X+\nabla_{\varphi X} \varphi X=\varphi[\varphi X, X]$. Thus any quasi-cosymplectic manifold satisfies the $\varphi$-condition.

Now, let $f: M \rightarrow M^{\prime}$ be a CR map among two quasi-cosymplectic manifolds. Then one gets

$$
\begin{aligned}
\beta_{f}(\varphi X, \varphi X)+\beta_{f}(X, X) & =\left\{\nabla_{X}^{\prime f} f_{*} X-f_{*} \nabla_{X} X+\nabla_{\varphi X}^{\prime f} f_{*} \varphi-f_{*} \nabla_{\varphi X} \varphi X\right\} \\
& =\left\{\nabla_{X}^{\prime f} f_{*} X+\nabla_{\varphi X}^{\prime f} \varphi^{\prime f} f_{*} X-f_{*}\left(\nabla_{X} X+\nabla_{\varphi X} \varphi X\right)\right\} .
\end{aligned}
$$

Consequently, since both $M$ and $M^{\prime}$ satisfy the $\varphi$-condition,

$$
\begin{aligned}
\beta_{f}(\varphi X, \varphi X)+\beta_{f}(X, X) & =\left(\varphi^{\prime f} f_{*}-f_{*} \varphi\right)[\varphi X, X] \\
& =\left(\varphi^{\prime}{ }^{f} f_{*}-f_{*} \varphi\right) g([\varphi X, X], \xi) \xi .
\end{aligned}
$$

Besides, $Y=\xi$ in (6.6) gives

$$
\varphi \nabla_{X} \xi=-\nabla_{\varphi X} \xi, \quad \nabla_{\xi} \xi=0 .
$$

Using (6.8), we have

$$
\begin{aligned}
g([\varphi X, X], \xi) & =g\left(\nabla_{\varphi X} X, \xi\right)-g\left(\nabla_{X} \varphi X, \xi\right) \\
& =-g\left(\nabla_{\varphi X} \xi, X\right)+g\left(\nabla_{X} \xi, \varphi X\right) \\
& =g\left(\varphi \nabla_{X} \xi, X\right)+g\left(\nabla_{X} \xi, \varphi X\right) \\
& =0 .
\end{aligned}
$$

Then, $\beta_{f}(\varphi X, \varphi X)+\beta_{f}(X, X)=0$, and by (5.1), we get: 
Theorem 6.1. Any $C R$ map $f: M \rightarrow M^{\prime}$ among two quasi-cosymplectic manifolds is Levi pluriharmonic.

Next, by (6.7) and (6.8), we get that any quasi-cosymplectic manifold satisfies

$$
\begin{aligned}
\operatorname{trace}(\varphi \nabla \xi) & =\sum_{\alpha=1}^{n}\left\{g\left(\varphi \nabla_{E_{\alpha}} \xi, E_{\alpha}\right)+g\left(\varphi \nabla_{\varphi E_{\alpha}} \xi, \varphi E_{\alpha}\right)\right\} \\
& =\sum_{\alpha=1}^{n}\left\{g\left(\varphi \nabla_{E_{\alpha}} \xi, E_{\alpha}\right)+g\left(\nabla_{E_{\alpha}} \xi, \varphi E_{\alpha}\right)\right\} \\
& =0 .
\end{aligned}
$$

Consequently, by Theorem 4.3, we have:

Theorem 6.2. A CR map $f: M \rightarrow M^{\prime}$ among two quasi-cosymplectic manifolds is a harmonic map if and only if $f_{*} \xi$ is a geodesic vector field. In particular, any $C R$ map $f: M \rightarrow M^{\prime}$ among two quasi-cosymplectic manifolds satisfying $f_{*} \xi=c \xi^{\prime}$, for some $c \in \mathbb{R}$, is a harmonic map.

\section{References}

[1] P. Baird and J. C. Wood, Harmonic morphisms between Riemannian manifolds, London Mathem. Society Monographs, Vol. 29, Oxford Science Publications, Clarendon Press, Oxford, 2003.

[2] E. Barletta, S. Dragomir, and H. Urakawa, Pseudoharmonic maps from nondegenerate CR manifolds to Riemannian manifolds, Indiana Univ. Math. J. 50 (2001), no. 2, 719746 .

[3] E. Barros and A. Romero, Indefinite Kähler manifolds, Math. Ann. 261 (1982), no. 1, $55-62$.

[4] A. Bejancu and K. L. Duggal, Real hypersurfaces of indefinite Kaehler manifolds, Int. J. Math. Math. Sci. 16 (1993), no. 3, 545-556.

[5] D. E. Blair, Riemannian Geometry of Contact and Symplectic Manifolds, Progress in Math. 203, Birkhäuser, Boston, Basel, Berlin, 2002.

[6] G. Calvaruso and D. Perrone, Contact pseudo-metric manifolds, Differential Geom. Appl. 28 (2010), no. 5, 615-634.

[7] M. Capursi, Quasicosymplectic manifolds, Rev. Roumaine Math. Pures Appl. 32 (1987), no. $1,27-35$.

[8] J. Davidov, Almost contact metric structures and twistor spaces, Houston J. Math. 29 (2003), no. 3, 639-673.

[9] S. Dragomir and D. Perrone, Harmonic Vector Fields: Variational Principles and Differential Geometry, Elsevier, Science Ltd, 2011.

[10] Levi harmonic maps of contact Riemannian manifolds, J. Geome. Anal. 24 (2014), no. 3, 1233-1275.

[11] B. Fuglede, Harmonic morphisms between semi-Riemannian manifolds, Ann. Acad. Sci. Fenn. Math. 21 (1996), no. 1, 31-50.

[12] Y. Ohnita, On pluriharmonicity of stable harmonic maps, J. London Math. Soc. (2) 35 (1987), no. 3, 563-568.

[13] Z. Olszak, On almost cosymplectic manifolds, Kodai Math. J. 4 (1981), no. 2, 239-250.

[14] D. Perrone, Classification of homogeneous almost cosymplectic three-manifolds, Differential Geom. Appl. 30 (2012), no. 1, 49-58. 
[15] _ Curvature of K-contact semi-Riemannian manifolds, Canad. Math. Bull. 57 (2014), no. 2, 401-412.

[16] Contact pseudo-metric manifolds of constant curvature and CR geometry, Results Math. 2014 Online first 2014, Springer Basel DOI 10.1007/s00025-014-0373-7.

[17] J. H. Rawnsley, f-structures, f-twistor spaces and harmonic maps, Geometry Seminar L. Bianchi, Lecture Notes in Math. 1164, 84-159, Springer-Verlag, 1985.

[18] K. Yano and S. Ishihara, Invariant submanifolds of an almost contact manifold, Kodai Math. Sem. Rep. 21 (1969), 350-364.

[19] K. Yano and M. Kon, Structures on Manifolds, World Scientific, Series in Pure Mathematics, vol. 3, 1984.

UNIVERSitÀ DEL SALENTO

Dipartimento di Matematica e Fisica "E. De Giorgi"

Via Provinciale Lecce-Arnesano

73100 LeCCE, ITALY

E-mail address: domenico.perrone@unisalento.it 